\title{
Interaction-Driven Metal-Insulator Transition in Strained Graphene
}

\author{
Ho-Kin Tang, ${ }^{1,2}$ E. Laksono, ${ }^{1,2}$ J. N. B. Rodrigues, ${ }^{1,2}$ P. Sengupta, ${ }^{1,3}$ F. F. Assaad, ${ }^{4}$ and S. Adam ${ }^{1,2,5}$ \\ ${ }^{1}$ Centre for Advanced 2D Materials, National University of Singapore, 6 Science Drive 2, Singapore 117546, Singapore \\ ${ }^{2}$ Department of Physics, Faculty of Science, National University of Singapore, 2 Science Drive 3, Singapore 117542, Singapore \\ ${ }^{3}$ School of Physical and Mathematical Sciences, Nanyang Technological University, 21 Nanyang Link, Singapore 637371, Singapore \\ ${ }^{4}$ Institut für Theoretische Physik und Astrophysik, Universität Würzburg, Am Hubland, D-97074 Würzburg, Germany \\ ${ }^{5}$ Yale-NUS College, 16 College Avenue West, Singapore 138527, Singapore
}

(Received 28 May 2015; published 30 October 2015)

\begin{abstract}
The question of whether electron-electron interactions can drive a metal to insulator transition in graphene under realistic experimental conditions is addressed. Using three representative methods to calculate the effective long-range Coulomb interaction between $\pi$ electrons in graphene and solving for the ground state using quantum Monte Carlo methods, we argue that, without strain, graphene remains metallic and changing the substrate from $\mathrm{SiO}_{2}$ to suspended samples hardly makes any difference. In contrast, applying a rather large_-but experimentally realistic_-uniform and isotropic strain of about $15 \%$ seems to be a promising route to making graphene an antiferromagnetic Mott insulator.
\end{abstract}

Over the past decade, graphene has established itself as a remarkable new material with superlative properties [1,2]. However, the early hopes to utilize it as a next-generation transistor have been dashed, mostly because graphene remains metallic - these prototypical Dirac fermions are immune to many of the conventional routes for driving twodimensional electron gases into an insulating state, including, for example, Anderson localization and percolation transitions (see, e.g., Ref. [3]). Other mechanisms for opening band gaps including hydrogenation [4], application of uniaxial strain [5], and forming nanoribbons [6] severely degrade graphene's mobility. Very recently, moiré heterostructures using graphene and hexagonal boron nitride have shown evidence of an insulating phase [7,8], although interpreting these results remains somewhat controversial [9-12].

In this Letter, we explore a different avenue to make graphene insulating, namely, utilizing the electron-electron interactions. Despite much study on the effects of interactions in graphene [13], it is surprising how much still remains to be understood. While it is clear that, without any electron-electron interactions, graphene should be a semimetal (SM), and that for very strong interactions it should be an insulating antiferromagnet (AFM), it remains unclear what one should expect for the real graphene material. For example, there are distinct claims in the literature that suspended graphene should be insulating, strongly metallic, and weakly metallic [14-16]. This discussion could have practical relevance, as it could be the basis for a low power Mott transistor [17].

In this work, we explore different ways of controlling the effective strength of electron-electron interactions in realistic graphene devices and propose how one can move around its phase diagram. In particular (and in contrast to what is widely assumed to be true $[2,13]$ ), we demonstrate that it is the nonuniversal, material-specific, and shortrange part of the electron-electron interactions that plays the dominant role in determining graphene's ground state. More interestingly, we conclude that the application of isotropic strain is considerably more efficient in approaching the SM-AFM phase transition than substrate manipulation, providing a new route for driving the system into the elusive Mott insulating phase that has yet to be observed experimentally.

The Hubbard model has served as a versatile paradigm to study interacting electrons on a lattice. It is defined as an effective model for electrons in partially filled narrow energy bands of a crystal's spectrum. While the canonical Hubbard model includes only on-site interactions, the effects of longer-range interactions are incorporated by a straightforward generalization of the two-body interaction term, described by the Hamiltonian

$$
\begin{aligned}
\hat{H}= & -t \sum_{\langle i j\rangle, \sigma}\left(\hat{c}_{\sigma i}^{\dagger} \hat{c}_{\sigma j}+\text { H.c. }\right)+\sum_{i} \hat{n}_{i \uparrow} \mathcal{V}_{i i} \hat{n}_{i \downarrow} \\
& +\frac{1}{2} \sum_{i \neq j} \sum_{\sigma, \sigma^{\prime}} \hat{n}_{i \sigma} \mathcal{V}_{i j} \hat{n}_{j \sigma^{\prime}},
\end{aligned}
$$

where $\hat{c}_{i \sigma}^{\dagger}\left(\hat{c}_{i \sigma}\right)$ creates (annihilates) an electron of spin $\sigma=\uparrow \downarrow$ at position $\mathbf{r}_{i}$ while $\hat{n}_{i \sigma}=\hat{c}_{i \sigma}^{\dagger} \hat{c}_{i \sigma}$ gives the density of electrons with spin $\sigma$ at position $\mathbf{r}_{i}$. The nearest neighbor hopping integral is identified by $t$, while $\mathcal{V}_{i j}$ stands for the interaction between electrons at sites $i$ and $j$. We note that a realistic description of graphene requires the parameters $\mathcal{V}_{i j}$ to be fixed in accordance with the spatial profile of the (partially screened) Coulomb interaction $\mathcal{V}(r)$ that results from the screening of the bare Coulomb interaction by electrons in energy bands other than the $\pi$ bands.

It is well established that the canonical Hubbard model [Eq. (1) with only on-site interactions, i.e., $\mathcal{V}_{i i}=U(>0)$ 


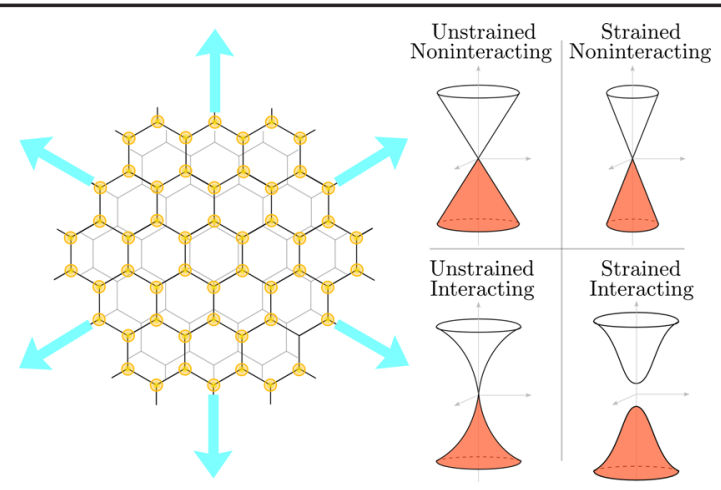

FIG. 1 (color online). Schematics of biaxially strained graphene (left panel). Representation of graphene's low-energy spectrum (right panels) of unstrained noninteracting and interacting graphene at half filling and biaxially strained noninteracting and interacting graphene at half filling.

and $\mathcal{V}_{i j}=0$ for all $i \neq j$ ] on the honeycomb lattice at half filling has a critical Hubbard on-site interaction parameter for the SM-AFM transition $U_{c}=(3.80 \pm 0.01) t$ [18-20]. Following various works based on $a b$ initio methods (see, e.g., Refs. [21,22]), it is generally agreed that $t=(2.7 \pm 0.2) \mathrm{eV}$. Estimates of the on-site interaction parameter $U$ for realistic experimental realizations of graphene vary widely in the literature [23-26], with values ranging from $U \approx 1$ to $10 \mathrm{eV}$ (where the lower estimates would suggest that graphene is metallic, while the higher estimates hint at it being insulating).

However, ignoring longer-range interactions in graphene is problematic, since the long-range tails of the Coulomb potential between Dirac fermions cannot be efficiently screened [27]. To address these Coulomb tails, it was recently conjectured [15] that the effects of nonlocal interactions can be mapped into the Hubbard model with an on-site interaction $\tilde{U}$ given by $\tilde{U} \approx U-V$, where $U \equiv \mathcal{V}_{i i}$ corresponds to the on-site interaction of the long-range Hubbard model, while $V \equiv \mathcal{V}_{i i+\delta}$ stands for the value of the Coulomb potential between electrons at two neighboring atoms on the honeycomb lattice. This effective $\tilde{U}$ would thus be the crucial factor determining graphene's phase. As we discuss below, our numerical calculation with the full long-range potential shows that this approximation is qualitatively correct but quantitatively inaccurate.

Here we study the possibility to drive graphene across the SM-AFM phase transition by substrate manipulation or application of biaxial (i.e., uniform and isotropic) strainsee Fig. 1. First, we must fix the long-range Hubbard model's parameters $\mathcal{V}_{i j}$. These are the crucial ingredients determining the ground state properties of the system, yet their real values are unknown. We use three representative methods to capture the full spatial profile of the partially screened Coulomb interaction for $p_{z}$ electrons in realistic graphene and choose $\mathcal{V}_{i j}$ accordingly. These methods will be discussed in detail below, but now we just introduce their names: Thomas-Fermi (TF), constrained random phase approximation (cRPA), and the quantum-chemistryPariser-Parr-Pople (QC-PPP) method. We then investigate the effect of biaxial strain and substrate manipulation on the partially screened Coulomb potential $\mathcal{V}(r)$. We find (see Fig. 2) that biaxial strain strongly modifies the $\mathcal{V}(r)$ close to $r=0$ (not affecting the long-range interactions), while changing the substrate (which changes both the dielectric screening [28] and the amount of disorder [2]) only weakly modifies the long-range tail of $\mathcal{V}(r)$. Finally, using quantum Monte Carlo techniques (finite temperature determinant quantum Monte Carlo and zero-temperature projective quantum Monte Carlo calculations), we simulate the ground state of the long-range half-filled Hubbard model (in the honeycomb lattice) with the $\mathcal{V}_{i j}$ obtained from $\mathcal{V}(r)$ and argue that, at least within the Thomas-Fermi approximation, an experimentally feasible [29,30] amount of strain would drive graphene into an interaction-driven insulating phase, which could be then measured in compressibility, transport, or scanning probe experiments.

We now detail the three methods that we use to estimate the partially screened Coulomb interactions that $p_{z}$ electrons feel. (These were chosen since they are very representative of the different approaches that have so far been used in the literature.) The cRPA method (see, e.g., Ref. [31]) was adapted to graphene by Wehling et al. [25]. In a systematic way, this method makes use of the electronic structure of graphene to compute, within the random phase approximation, the polarization function $P_{r}$ associated with all the interaction events other than those involving two electrons from the $\pi$ bands. Then, the effective (partially) screened Coulomb interaction felt by the $p_{z}$ electrons is given by $\mathcal{V}(r)=\left[1-V_{\text {bare }}(r) P_{r}\right]^{-1} V_{\text {bare }}(r)$, where $V_{\text {bare }}(r)$ stands for the bare Coulomb potential. The accuracy of this method has long been debated in the literature (see, e.g., Ref. [32]), and its results are often difficult to interpret physically. For graphene, the fact that the Dirac band spans a broad energy window further complicates the application of the cRPA
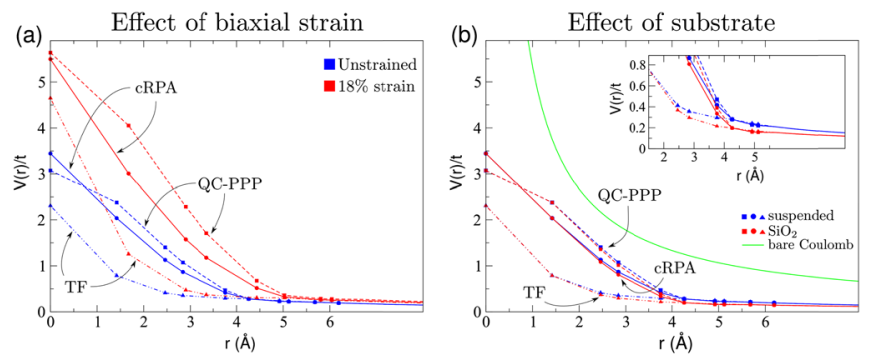

FIG. 2 (color online). Effect of biaxial strain (left panel) and substrate (right panel) on the partially screened Coulomb interaction. We use three representative models: cRPA, circles and full curves; QC-PPP, squares and dashed curves; and TF, triangles and dot-dashed curves. (a) Suspended graphene both unstrained and subject to $18 \%$ biaxial strain. (b) Unstrained graphene both suspended and deposited on $\mathrm{SiO}_{2}$ compared to the bare Coulomb potential. 
formalism. Notwithstanding these difficulties, the cRPA remains the best numerical technique at our disposal to determine the $\mathcal{V}_{i j}$ for graphene. In this Letter, we use the cRPA results previously obtained in Ref. [25], which compute $U, V$, and $t$ for biaxial strains up to $12 \%$. In this range of strains, all these parameters show a linear behavior. In order to obtain the cRPA values of $U, V$, and $t$ for up to $18 \%$ strain [see Fig. 2(a)], we have assumed that this linear behavior remains unchanged, extracting $U, V$, and $t$ from a linear fit to the numerical results of Ref. [25].

The QC-PPP method (see, e.g., Vergés et al. [33]) works by using $a b$ initio Hartree-Fock and post-Hartree-Fock techniques to solve for the ground state energy of molecules comprising a small number of benzene rings. These energies are then compared to an exact diagonalization of the longrange Hubbard model where the Ohno interpolation formula $\mathcal{V}(r)=U / \sqrt{1+\left(U r / e^{2}\right)^{2}}$ is assumed for the Coulomb interaction. The $\mathcal{V}(0)=U$ is a free parameter that is fixed by requiring the minimization of the root-mean square of the ground state energy of the $a b$ initio calculations and that of the long-range Hubbard model. The QC-PPP values of $U$ and $V$ used in this Letter were extracted from Ref. [33], which calculates $\mathcal{V}(r)$ for the phenalenyl $\left(3 \mathrm{H}-\mathrm{C}_{13} \mathrm{H}_{9}\right)$ molecule. This method gives an upper bound for the Hubbard $U$ in graphene, since larger molecules would have more screening and reduced $\mathcal{V}(r)$. Both the validity of the Ohno interpolation and the extrapolation to larger system sizes give some reasons for caution. It has nonetheless proven extremely useful for small $\pi$-conjugated planar polycyclic aromatic hydrocarbons comprising tens of atoms such as anthracene and polyacenes [33,34].

Finally, inspired by the work of Jung and MacDonald [26], we have constructed a Thomas-Fermi model to account for the screening of higher energy bands in graphene. Within the Thomas-Fermi screening approximation, the on-site interaction $U$ is given by

$$
U=\frac{e^{2}}{4 \pi \epsilon} \int d^{3} \mathbf{r}_{1} d^{3} \mathbf{r}_{2}\left|\phi\left(\mathbf{r}_{1}\right)\right|^{2} \frac{e^{-k_{0}\left|\mathbf{r}_{1}-\mathbf{r}_{2}\right|}}{\left|\mathbf{r}_{1}-\mathbf{r}_{2}\right|}\left|\phi\left(\mathbf{r}_{2}\right)\right|^{2},
$$

while the Coulomb interaction between two $\pi$-bands' electrons positioned at neighboring atoms (distance $\delta$ ) $\mathrm{V}$ is given by

$V=\frac{e^{2}}{4 \pi \epsilon} \int d^{3} \mathbf{r}_{1} d^{3} \mathbf{r}_{2}\left|\phi\left(\mathbf{r}_{1}+\boldsymbol{\delta}\right)\right|^{2} \frac{e^{-k_{0}\left|\mathbf{r}_{1}-\mathbf{r}_{2}\right|}}{\left|\mathbf{r}_{1}-\mathbf{r}_{2}\right|}\left|\phi\left(\mathbf{r}_{2}\right)\right|^{2}$.

Here, $\phi(r)$ stands for the $p_{z}$ orbital wave function (which we approximate by that of atomic hydrogen). The free parameter $k_{0}$ in Eqs. (2) and (3) is fixed by requiring that the hopping integral

$t=\int d^{3} \mathbf{r} \phi^{*}(\mathbf{r}+\boldsymbol{\delta})\left[-\frac{\hbar^{2} \nabla^{2}}{2 m}+\frac{e^{2}}{4 \pi \epsilon} \sum_{i} \frac{e^{-k_{0}\left|\mathbf{r}-\mathbf{R}_{i}\right|}}{\left|\mathbf{r}-\mathbf{R}_{i}\right|}\right] \phi(\mathbf{r})$ is equal to the literature-accepted value of $t_{0}=2.7 \mathrm{eV}$ [21]. In parallel with what we do for the other two methods, we then interpolate between $\mathcal{V}_{i j}$ 's short-range values $U$ and $V$ and the long-range tail of $\mathcal{V}_{i j}$ (see below). The procedure used to compute $\mathcal{V}_{i j}$ of biaxially strained graphene is similar to that discussed earlier [34].

The computationally demanding method employed prevents us from simulating large size systems. In particular, one must include the effect of the surrounding electrons, since their interband polarizability contributes at all length scales [2], thus modifying the effective dielectric constant from $1 / r$ to $1 /\left[r\left(1+\pi r_{s} / 2\right)\right]$, where $r_{s}=2 e^{2} /\left[\left(\kappa_{a}+\right.\right.$ $\left.\left.\kappa_{b}\right) \hbar v_{F}\right]$ is the effective fine structure constant (where $\kappa_{a}$ and $\kappa_{b}$ are the dielectric constants above and below the graphene flake, respectively). The presence of disorder in the substrate can also be accounted for by introducing a modified screening function (see, e.g., Ref. [35]). The full profile of the partially screened Coulomb interaction is obtained by interpolating between the short-range results at first neighbor distance and the long-range tail (assumed to start at the fourth neighbor).

As we can see in Fig. 2, the short-range part of the partially screened Coulomb interactions $\mathcal{V}(r)$ is strongly affected by biaxial strain (left panel), while its long-range tails are nearly insensitive to strain. Manipulating the substrate has a very weak effect on the long-range tails of the partially screened Coulomb interactions (right panel).

With the electron-electron interaction profiles of Fig. 2, we have fixed the long-range Hubbard model's parameters $\mathcal{V}_{i j}$ and explored its ground state using auxiliary field quantum Monte Carlo simulations (made possible by recent works $[16,36,37])$ - a numerically exact method for investigating strongly correlated systems. In this Letter, we use different implementations of the auxiliary field quantum Monte Carlo technique: finite temperature determinant quantum Monte Carlo (DQMC) calculations, whose correlation functions are given by

$$
\langle\hat{O}\rangle=\frac{1}{Z} \operatorname{Tr}\left[\hat{O} e^{\beta H}\right]=\frac{1}{Z} \int \mathcal{D}\left[\phi_{i, \tau}\right] e^{-S\left[\phi_{i, \tau}\right]} \hat{O}\left[\phi_{i, \tau}\right],
$$

(we refer the reader to Ref. [38] for details), and zerotemperature projective quantum Monte Carlo (PQMC) (for details, see, e.g., Ref. [39]), where the correlation functions are given by

$$
\langle\hat{O}\rangle=\frac{\left\langle\Phi_{0}|\hat{O}| \Phi_{0}\right\rangle}{\left\langle\Phi_{0} \mid \Phi_{0}\right\rangle}=\lim _{\Theta \rightarrow \infty} \frac{\left\langle\Psi_{T}\left|e^{-\Theta \hat{H} / 2} \hat{O} e^{-\Theta \hat{H} / 2}\right| \Psi_{T}\right\rangle}{\left\langle\Psi_{T}\left|e^{-\Theta \hat{H}}\right| \Psi_{T}\right\rangle} .
$$

In both cases, we use a Hubbard-Stratonovich transformation to convert the interaction term into a noninteracting term coupled to an auxiliary field. This transformation enables us to treat Hubbard models with nonlocal electron interactions, provided that the long-range interaction gives rise to a transformation matrix that is positive definite (a nonpositive definite transformation matrix corresponds to a diverging auxiliary field). 
In particular, we find that the transformation matrix for the case where the $\mathcal{V}_{i j}$ is obtained from the QC-PPP method is not positive definite. This is a direct consequence of the interpolation scheme mentioned above, which renders the off-diagonal matrix elements associated with the nonlocal interaction comparable with the diagonal elements associated with the local interactions. As a result, we could not use quantum Monte Carlo calculations to simulate the QC-PPP model.

In the DQMC, we used inverse temperature $\beta=(1 / T)$ in Eq. (5) between 24 and 36, which is sufficient to probe the low-energy behavior of the system. In the PQMC, we chose the Hartree-Fock state as our trial wave function, $\left|\Psi_{T}\right\rangle$, using $\Theta t=40$ [see Eq. (6)] to project the wave function onto the ground state. We made use of the scaling behavior of the antiferromagnetic structure factor $\left(S_{\mathrm{AFM}}\right)$ to estimate the magnetic state of the system:

$$
S_{\mathrm{AFM}}=\frac{1}{L^{2}} \sum_{i, j}\left[\left\langle m_{i A} m_{j A}\right\rangle+\left\langle m_{i B} m_{j B}\right\rangle\right],
$$

where $m_{i C}$ stands for the magnetization of the site located in the atom of sublattice $C=A, B$ of the unit cell $\mathbf{r}_{i}$, while $L^{2}$ is the number of unit cells (i.e., $N=2 L^{2}$ sites). The system's AFM order parameter is given by $m_{\mathrm{AFM}}=\sqrt{S_{\mathrm{AFM}} /\left(2 L^{2}\right)}$. We have simulated lattice sizes between $L=6$ and $L=15$. In order to take finite size effects into account, we utilize $\tilde{m}_{\mathrm{AFM}}=m_{\mathrm{AFM}} L^{\beta / \nu}$, where we use the critical exponents $\beta / \nu \approx 0.9$ (obtained from the best data collapse in Ref. [36]), compatible with the Gross-Neveu universality class $[20,40]$.

Figure 3 shows the dependence of $m_{\mathrm{AFM}}$ and $\tilde{S}_{\mathrm{AFM}}$ with the system size. For unstrained graphene, both the cRPA and TF methods show $\tilde{S}_{\mathrm{AFM}}$ decreasing with system size (and $m_{\mathrm{AFM}}$ extrapolating to zero in the thermodynamic limit $L \rightarrow \infty$ ), indicating that, without strain, suspended graphene is metallic (in agreement with experimental observations). However, most interestingly, with $18 \%$ biaxial strain, the TF model shows $\tilde{S}_{\mathrm{AFM}}$ increasing with the system size (with $m_{\mathrm{AFM}}$ extrapolating to a nonzero value when $L \rightarrow \infty$ ), indicating an antiferromagnetic Mott insulator in the thermodynamic limit. This corresponds to an interaction-driven gap of $\Delta=(0.55 \pm 0.05) \mathrm{eV}$, comparable to estimates in Ref. [41] obtained by hybrid density functional calculations (Hartree-Fock exchange hybridized with generalized gradient approximation for the exchange correlation) that do not accurately treat the effects of electron correlations. Moreover, within the Thomas-Fermi method, our QMC calculations find a critical strain of $\eta_{c} \approx 0.15$. Notice that, in this case, $\tilde{U}=U-V=3.4 t<U_{c}$, demonstrating that the suggestion by Ref. [15] for mapping the long-range Hubbard model for graphene into an effective on-site Hubbard model is quantitatively inaccurate.

Although the TF method has no adjustable parameters, it assumes that the Coulomb interaction between $p_{z}$ electrons

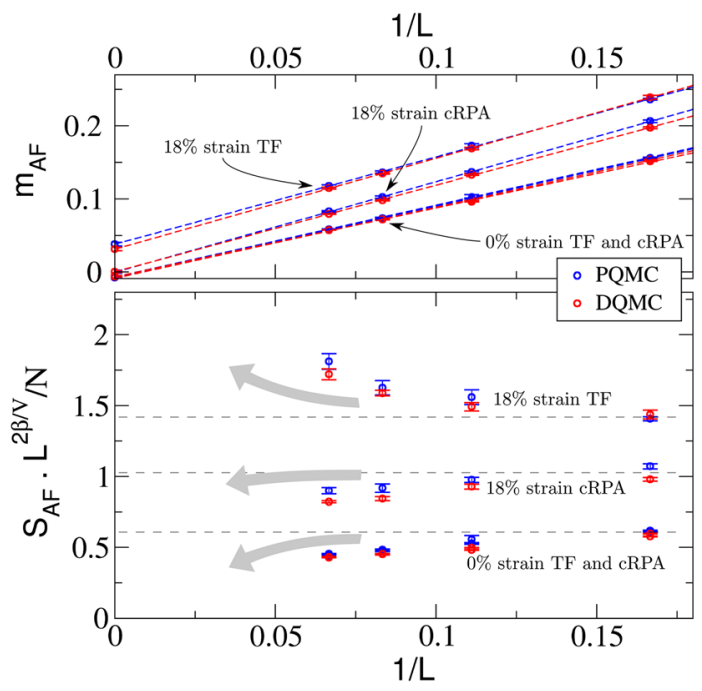

FIG. 3 (color online). Antiferromagnetic (AFM) order parameter $m_{\mathrm{AFM}}=\sqrt{S_{\mathrm{AFM}} /\left(2 L^{2}\right)}$ (top) and scaled antiferromagnetic structure factor $S_{\mathrm{AFM}} L^{2 \beta / \nu} / N$ (bottom) in terms of the inverse system size. We have used both projective quantum Monte Carlo (blue) and determinant quantum Monte Carlo (red) calculations to study the phase of graphene subject to: $18 \%$ biaxial strain within the Thomas-Fermi model (upper points); 18\% biaxial strain within the constrained RPA model (middle points); and 0\% strain within both the Thomas-Fermi and the constrained RPA models (lower points). We could not simulate the quantumchemistry-Pariser-Parr-Pople model with auxiliary field quantum Monte Carlo calculations, since its partially screened Coulomb potential gives rise to diverging auxiliary fields.

on the same atom and between neighboring atoms is screened in the same way [26]. This assumption slightly overestimates the ratio $U / V$, giving a smaller critical strain $\eta_{c}$ for the SM-AFM transition. On the other hand, the canonical cRPA method ignores bandwidth and low-energy spectral weight reduction originating from integrating out the high-energy bands [32]. This gives rise to artificially weak partially screened Coulomb interactions, resulting in an overestimation of the critical strain $\eta_{c}$. Because of finite sizes, the PPP model overestimates the value of $U$ and $V$, and the Ohno interpolation underestimates their difference. However, looking at the three models together, we therefore conclude that the profile of the Coulomb potential for realistic graphene lies somewhere in between the TF and cRPA estimates. The TF model gives a maximum Mott gap of more than an order of magnitude larger than room temperature, and this value sets the upper bound for experiments.

In summary, using the best available models in the literature to estimate the effective Coulomb interaction between $p_{z}$ electrons in graphene, we have employed quantum Monte Carlo simulations to explore graphene's phase diagram in response to parameters that can be changed experimentally. We have found, surprisingly, that manipulating the short-range part of the effective Coulomb potential (i.e., the nonuniversal and material-specific 
component) is the crucial factor in determining the phase of graphene. Most importantly, we show that the application of experimentally realistic amounts of isotropic strain is a promising route to cross the SM-AFM quantum phase transition and to observe a strongly correlated state in this otherwise weakly interacting material.

H.-K. T. thanks Miguel Costa for helpful insights. S. A. thanks Garnet Chan, Jeil Jung, and Timo Lähde for fruitful discussions. H.-K. T., J. N. B. R., and S. A. are supported by the National Research Foundation of Singapore under its Fellowship program (NRF-NRFF2012-01) and by the Singapore Ministry of Education and Yale-NUS College through Grant No. R-607-265-01312. P. S. is supported by the Ministry of Education of Singapore through Grant No. MOE2011-T2-1-108. F. F. A. acknowledges the financial support from DFG Grant No. AS120/9-1. We acknowledge the use of the CA2DM and GRC high-performance computing facilities.

[1] A. H. Castro Neto, F. Guinea, N. M. R. Peres, K. S. Novoselov, and A. K. Geim, Rev. Mod. Phys. 81, 109 (2009).

[2] S. Das Sarma, S. Adam, E. H. Hwang, and E. Rossi, Rev. Mod. Phys. 83, 407 (2011).

[3] M. S. Fuhrer and S. Adam, Nature (London) 458, 38 (2009).

[4] D. Elias et al., Science 323, 610 (2009).

[5] Z. H. Ni, T. Yu, Y. H. Lu, Y. Y. Wang, Y. P. Feng, and Z. X. Shen, ACS Nano 2, 2301 (2008).

[6] M. Y. Han, B. Özyilmaz, Y. Zhang, and P. Kim, Phys. Rev. Lett. 98, 206805 (2007).

[7] B. Hunt, J. D. Sanchez-Yamagishi, A. F. Young, M. Yankowitz, B. J. LeRoy, K. Watanabe, T. Taniguchi, P. Moon, M. Koshino, P. Jarillo-Herrero, and R. C. Ashoori, Science 340, 1427 (2013).

[8] L. A. Ponomarenko, A. K. Geim, A. A. Zhukov, R. Jalil, S. V. Morozov, K. S. Novoselov, I. V. Grigorieva, E. H. Hill, V. V. Cheianov, V. I. Fal'ko, K. Watanabe, T. Taniguchi, and R. V. Gorbachev, Nat. Phys. 7, 958 (2011).

[9] J. Jung, A. M. DaSilva, A. H. MacDonald, and S. Adam, Nat. Commun. 6, 6308 (2015).

[10] B. Amorim, A. Cortijo, F. de Juan, A. G. Grushin, F. Guinea, A. Gutiérrez-Rubio, H. Ochoa, V. Parente, R. Roldán, P. San-José, J. Schiefele, M. Sturla, and M. A. H. Vozmediano, arXiv:1503.00747.

[11] F. Guinea and M. I. Katsnelson, Phys. Rev. Lett. 112, 116604 (2014).

[12] S. Das Sarma, E. H. Hwang, and Q. Li, Phys. Rev. B 85, 195451 (2012).

[13] V. N. Kotov, B. Uchoa, V. M. Pereira, F. Guinea, and A. H. Castro Neto, Rev. Mod. Phys. 84, 1067 (2012).

[14] J. E. Drut and T. A. Lähde, Phys. Rev. Lett. 102, 026802 (2009).

[15] M. Schüler, M. Rösner, T. O. Wehling, A. I. Lichtenstein, and M. I. Katsnelson, Phys. Rev. Lett. 111, 036601 (2013).

[16] M. V. Ulybyshev, P. V. Buividovich, M. I. Katsnelson, and M. I. Polikarpov, Phys. Rev. Lett. 111, 056801 (2013).
[17] M. Nakano, K. Shibuya, D. Okuyama, T. Hatano, S. Ono, M. Kawasaki, Y. Iwasa, and Y. Tokura, Nature (London) 487, 459 (2012).

[18] S. Sorella, Y. Otsuka, and S. Yunoki, Sci. Rep. 2, 992 (2012).

[19] F. F. Assaad and I. F. Herbut, Phys. Rev. X 3, 031010 (2013).

[20] F. Parisen Toldin, M. Hohenadler, F. F. Assaad, and I. F. Herbut, Phys. Rev. B 91, 165108 (2015).

[21] S. Reich, J. Maultzsch, C. Thomsen, and P. Ordejón, Phys. Rev. B 66, 035412 (2002).

[22] J. Jung and A. H. MacDonald, Phys. Rev. B 87, 195450 (2013).

[23] O. V. Yazyev, Phys. Rev. Lett. 101, 037203 (2008).

[24] S. Dutta, S. Lakshmi, and S. K. Pati, Phys. Rev. B 77, 073412 (2008).

[25] T. O. Wehling, E. Şaşığlu, C. Friedrich, A. I. Lichtenstein, M. I. Katsnelson, and S. Blügel, Phys. Rev. Lett. 106, 236805 (2011).

[26] J. Jung and A. H. MacDonald, Phys. Rev. B 84, 085446 (2011).

[27] S. Adam, E. H. Hwang, V. M. Galitski, and S. Das Sarma, Proc. Natl. Acad. Sci. U.S.A. 104, 18392 (2007).

[28] C. Jang, S. Adam, J.-H. Chen, E. D. Williams, S. Das Sarma, and M. S. Fuhrer, Phys. Rev. Lett. 101, 146805 (2008).

[29] K. S. Kim, Y. Zhao, H. Jang, S. Y. Lee, J. M. Kim, K. S. Kim, J.-H. Ahn, P. Kim, J.-Y. Choi, and B. H. Hong, Nature (London) 457, 706 (2009).

[30] V. M. Pereira, A. H. Castro Neto, and N. M. R. Peres, Phys. Rev. B 80, 045401 (2009).

[31] F. Aryasetiawan, K. Karlsson, O. Jepsen, and U. Schönberger, Phys. Rev. B 74, 125106 (2006).

[32] M. Casula, P. Werner, L. Vaugier, F. Aryasetiawan, T. Miyake, A. J. Millis, and S. Biermann, Phys. Rev. Lett. 109, 126408 (2012).

[33] J. A. Vergés, E. SanFabián, G. Chiappe, and E. Louis, Phys. Rev. B 81, 085120 (2010).

[34] Given the lack of published QC-PPP computations for strained aromatic hydrocarbon molecules, we have obtained the long-range Hubbard model parameters for graphene subject to $18 \%$ biaxial strain (i.e., $\eta=0.18$ ) from the unstrained $\mathcal{V}(r)$ computed by Ref. [33] but with $r \rightarrow(1+\eta) r$, while the hopping parameter $t$ was obtained from the results of Pereira and co-workers: $t \rightarrow t_{0} e^{-3.37 \eta}$ (see Ref. [30] for details).

[35] S. Adam, S. Jung, N. N. Klimov, N. B. Zhitenev, J. A. Stroscio, and M. D. Stiles, Phys. Rev. B 84, 235421 (2011).

[36] M. Hohenadler, F. Parisen Toldin, I. F. Herbut, and F. F. Assaad, Phys. Rev. B 90, 085146 (2014).

[37] R. C. Brower, C. Rebbi, and D. Schaich, Proc. Sci., Lattice2011 (2011) 056.

[38] R. R. d. Santos, Braz. J. Phys. 33, 36 (2003).

[39] F. F. Assaad, Quantum Monte Carlo Methods on Lattices: The Determinantal Approach (John von Neumann Institute for Computing, Jülich, 2002), pp. 99-156.

[40] I. F. Herbut, Phys. Rev. Lett. 97, 146401 (2006).

[41] S.-H. Lee, S. Kim, and K. Kim, Phys. Rev. B 86, 155436 (2012). 